\title{
Decreased Interaction of Raf-1 with Its Negative Regulator Spry2 as a Mechanism for Acquired Drug Resistance
}

\author{
Jun-Ho Ahn', Yun-Ki Kim ${ }^{1}$ and Michael Lee,* \\ ${ }^{1}$ Division of Life Sciences, College of Natural Sciences, University of Incheon, Incheon 406-772, Republic of Korea
}

\begin{abstract}
Experiments were carried out to determine the role of Raf-1 kinase in the development of drug resistance to paclitaxel in $\mathrm{v}-\mathrm{H}$-ras transformed NIH 3T3 fibroblasts (Ras-NIH 3T3). We established a multidrug-resistant cell line (Ras-NIH 3T3/Mdr) from Ras-NIH 3T3 cells by stepwise increases in paclitaxel. Drug sensitivity assays indicated that the $I_{50}$ value for drug-resistant Ras-NIH 3T3/Mdr cells was more than $1 \mu \mathrm{M}$ paclitaxel, 10- or more-fold higher than for the parental Ras-NIH 3T3 cells. Western blot and RT-PCR analysis showed that the drug efflux pump a P-glycoprotein were highly expressed in Ras-NIH 3T3/Mdr cells, while not being detectable in Ras-NIH 3T3 cells. Additionally, verapamil, which appears to inhibit drug efflux by acting as a substrate for P-glycoprotein, completely reversed resistance to paclitaxel in Ras-NIH 3T3/Mdr cell line, indicating that resistance to paclitaxel is associated with overexpression of the multidrug resistance gene. Interestingly, Ras-NIH 3T3/Mdr cells have higher basal Raf-1 activity compared to Ras-NIH 3T3 cells. Unexpectedly, however, the colocalization of Raf-1 and its negative regulator Spry2 was less observed in cytoplasm of Ras-NIH 3T3/Mdr cells due to translocation of Spry2 around the nucleus in the perinuclear zone, implying that Raf-1 may be released from negative feedback inhibition by interacting with Spry2. We also showed that shRNAmediated knockdown of Raf-1 caused a moderate increase in cell susceptibility to paclitaxel. Thus, the results presented here suggest that a Raf-1-dependent pathway plays an important role in the development of acquired drug-resistance.
\end{abstract}

Key Words: Paclitaxel, Raf-1, MDR, Chemotherapy, Spry2

\section{INTRODUCTION}

The resistance to the drug over time limits the efficacy of paclitaxel in anticancer therapy (McGrogan et al., 2008), although paclitaxel is an antineoplastic agent with proven efficacy in the treatment of breast, ovarian, lung, and colon cancers (Haldar et al., 1996; Lee et al., 1997). The mechanisms by which cancer cells become multidrug resistant is thought to be correlated predominantly with the overexpression of Pglycoprotein efflux pump (Callaghan and Higgins, 1995; Yusa and Tsuruo, 1989; Gottesman, 2002). Thus, the development of compounds that block P-glycoprotein-mediated efflux has been the conventional approach used to overcome multidrugresistance (MDR) (Wu et al., 2008). However, the exact mechanisms responsible for the increase in P-glycoprotein levels following chemotherapeutic therapy remain to be established. Selective expression of protein kinase $\mathrm{C}$ isozymes has been known to be correlated with MDR (Blobe et al., 1993; Gupta et al., 1996). Other works have revealed a link between sphingomyelinase activity and MDR (Jaffrezou et al., 1991). On the other hand, the available data on the role of Raf- 1 in paclitax-

www.biomolther.org

Open Access DOI: 10.4062/biomolther.2011.19.2.174

pISSN: 1976-9148 elSSN: 2005-4483

Copyright $\odot 2011$ The Korean Society of Applied Pharmacology el-induced acquired drug-resistance are still controversial, although previous researches have indicated that Raf-1 overexpression might be related to the MDR development in human cancer cell lines in vitro (Weinstein-Oppenheimer et al., 2001; Davis et al., 2003; Dong et al., 2009)

Raf- 1 , a cytoplasmic serine/threonine protein kinase, is activated by an interaction with Ras-GTP, which recruits Raf-1 to the plasma membrane and induces a conformation change that relieves an inhibition imposed by the $\mathrm{N}$ terminus on the catalytic domain (Marshall, 1995). Regarding the mechanism responsible for Raf-1 inactivation, Yeung and colleagues identified the suppressing effect of the Raf-1 kinase inhibitory protein (RKIP) on the activation status of the Raf-1/MEK/ ERK pathways (Yeung et al., 1999). RKIP involvement in the reversal of tumor cell resistance to chemotherapeutic agents has also been reported previously (Chatterjee et al., 2004; Jazirehi et al., 2004). Other more potent inhibitor of the Raf-1 kinase activation is the Sprouty (Yusoff et al., 2002; Sasaki et al., 2003), which was first identified in a genetic screen in Drosophila (Hacohen et al., 1998). Our previous reports suggested that the enhancement of Raf-1 kinase activity by Spry2

Received Dec 6, 2010 Revised Feb 9, 2011 Accepted Mar 2, 2011

*Corresponding Author
E-mail: mikelee@incheon.ac.kr
Tel: +82-32-835-8247, Fax: +82-32-835-0763 
knockdown was associated with high sensitivity to paclitaxel in Ras-NIH 3T3 cells (Ahn et al., 2009).

The present research was performed to investigate the correlation of Raf-1 kinase activity with the MDR development of Ras-NIH 3T3 cells. Our findings suggest that the enhancement of Raf-1 kinase activity, which occurs in parallel with the decrease in the interaction with Spry2, may contribute to the development of acquired resistance in Ras-NIH 3T3 cells. Furthermore, we explored the reversal effect of Raf-1 shRNA transfection on the multidrug resistance, with the aim of gaining a promising therapy to reverse the tumor MDR efficiently.

\section{MATERIALS AND METHODS}

\section{Antibodies and reagents}

Mouse monoclonal anti-Raf (E-10), polyclonal anti-ERK, and anti-Bcl-2 were obtained from Santa Cruz Biotechnology (Santa Cruz, CA), while anti-cleaved PARP, anti-phosphoMEK (Ser217/221), and anti-phospho-ERK (Thr202/Tyr204) were from Cell Signaling Technology (Denvers, MA). A polyclonal anti-spry2 was purchased from Millipore Co. (Billerca, MA). Protein A-agarose was from Roche Molecular Biochemicals (Indianapolis, IN). Dulbecco's modified Eagle's medium (DMEM), fetal calf serum and penicillin-streptomycin were purchased from GIBCO-Invitrogen (Carlsbad, CA). Reagents for SDS-polyacrylamide gel electrophoresis were from BioRad (Hercules, CA). Paclitaxel and verapamil were obtained from Sigma (St. Louis, MO).

\section{Mammalian cell culture, transient transfection and chemi- cal treatment}

Ras-NIH 3T3 cells, which show morphologically transformed foci of cells with the characteristics of crisscrossed margins, piling up properties and invasiveness (Lee et al., 2009), were maintained at $37^{\circ} \mathrm{C}$ in DMEM supplemented with $10 \%$ FCS, penicillin-streptomycin, and glutamine. For experimental purposes, cells were cultured in $60-\mathrm{mm}$ tissue culture dishes until they reached $-80 \%$ confluency. For Raf- 1 knockdowns, short hairpin RNA (shRNA) constructs against Raf-1 (catalog number TR516793) were purchased from OriGene Technologies, Inc. (Rockville, MD, USA). The targeted sequences were: TGTGACATCTGTCAGAAGTTCCTGCTAAA (TI593078). Raf-1 shRNA was transfected into cells using Lipofectamine 2000 (Invitrogen) in Opti-minimal essential medium I medium (Invitrogen) according to the manufacturer's protocol. After $24 \mathrm{~h}$, the transfected cells were treated with chemical. Paclitaxel was dissolved in DMSO and freshly diluted for each experiment. DMSO concentrations were less than $0.1 \%$ in all experiment.

\section{Establishment of paclitaxel-resistant v-H-ras-transformed cell line}

Paclitaxel-resistant Ras-NIH 3T3/Mdr cells were derived from Ras-NIH 3T3 cells by stepwise increased concentrations of paclitaxel. Briefly, paclitaxel was first added to the parental Ras-NIH 3T3 cells to a final concentration of $100 \mathrm{nM}$, and the cells were incubated at $37^{\circ} \mathrm{C}$ for 2 days. Then the medium was changed to fresh one without paclitaxel and cells were cultured until the cells were again almost $80 \%$ confluent. The concentration of paclitaxel was then increased by $50 \mathrm{nM}$ increments until a concentration of $800 \mathrm{nM}$ was reached. This cyclic treatment was repeated over a period of 18 weeks. The new Ras-NIH 3T3/Mdr cell line that grew at the maximum concentration of paclitaxel was stored for further analyses. For maintenance of paclitaxel-resistant cells, $100 \mathrm{nM}$ paclitaxel was added into the normal medium. Before experimental use, Ras-NIH 3T3/Mdr cells were maintained in a paclitaxel-free culture medium and subcultured at least three times.

\section{Cell growth assay}

The cell proliferation reagent WST-1 was used for the quantitative determination of cellular proliferation and activation (Roche Molecular Biochemicals, Germany). For proliferation assays, the cells were plated in quadruplicates into 96well microliter plates (Costar, Cambridge, MA) at $5 \times 10^{3}$ cells/ well and then treated with paclitaxel at $37^{\circ} \mathrm{C}$ in a humidified $5 \% \mathrm{CO} 2 / 95 \%$ air incubator. After incubation for 2 or 3 days, the cells were incubated for additional $4 \mathrm{~h}$ in the presence of WST-1 labeling mixture (10 $\mu \mathrm{l}$ per well). The absorbance of the samples against a background control (medium alone) as a blank was measured at $450 \mathrm{~nm}$ using a microliter plate (ELISA) reader (Molecular Devices, Sunnyvale. CA).

\section{RNA extraction and RT-PCR}

Twenty-four hours after plating of $1 \times 10^{6}$ Ras-NIH 3T3 or Ras-NIH 3T3/Mdr cells, total RNA was purified with RNeasy mini kit (QIAGEN, Valencia, CA). Complementary DNA (cDNA) was made by reverse-transcription (RT) of $1 \mu \mathrm{g}$ each total RNA using OneStep RT-PCR Kit (QIAGEN). The primer sets were 5'-tgcttatggatcccagagtgac-3' and 5'-ttggtgaggatctctccggct-3' for $m d r 1$; 5'-agtgaaaggggctacagggt-3' and 5'-aacttctgctcccgagtcaa-3' for $m d r 3$. Each of the amplified PCR products was determined by electrophoresis on an $1.5 \%$ agarose gel.

\section{Preparation of cell lysates and immunoblot analysis}

The treatments of cells were carried out at $37^{\circ} \mathrm{C}$ in serumfree medium as described in the figure legends. Following treatment, whole cell lysates were prepared as follows. Cells were washed twice with ice-cold phosphate-buffered saline (PBS), and harvested by scraping the cells into lysis buffer (20 mM Tris, pH 8.0, $150 \mathrm{mM} \mathrm{NaCl}, 1 \%$ Triton X-100, 2 mM EDTA, $1 \mathrm{mM}$ phenylmethylsulfonyl fluoride, $20 \mu \mathrm{g} / \mathrm{ml}$ aprotinin, 10 $\mu \mathrm{g} / \mathrm{ml}$ leupeptin, $20 \mathrm{mM} \beta$-glycerophosphate and $2 \mathrm{mM}$ sodium fluoride). Cell lysates were clarified by centrifugation at $15,000 \times \mathrm{g}$ for 10 minutes at $4^{\circ} \mathrm{C}$, and lysate protein concentrations were determined with a BCA protein assay reagent kit as described by the manufacturer (Pierce; Rockford, IL). For immunoblotting, immunoprecipitates or whole cell lysates were denatured in Laemmli sample buffer, and resolved by SDS-polyacrylamide gel. The proteins were transferred to nitrocellulose, and immunoblot analysis was performed using appropriate primary antibodies. Immune complexes on nitrocellulose were detected by the ECL-Plus chemiluminescent system (Amersham Pharmacia Biotech, Piscataway, NJ). Fluorescent images were captured using KODAK Image Station 4000R (Carestream Health, Inc., Rochester, NY). Bands were quantified using Kodak Molecular Imaging software, version 4.5.0 (Carestream Health, Inc.).

\section{In vitro Raf-1 kinase activity assay}

Raf-1 proteins were specifically immunoprecipitated from whole cell lysates using $1 \mu \mathrm{g}$ monoclonal anti-Raf- 1 antibody directed against the carboxy-terminal domain. After incubation 
A

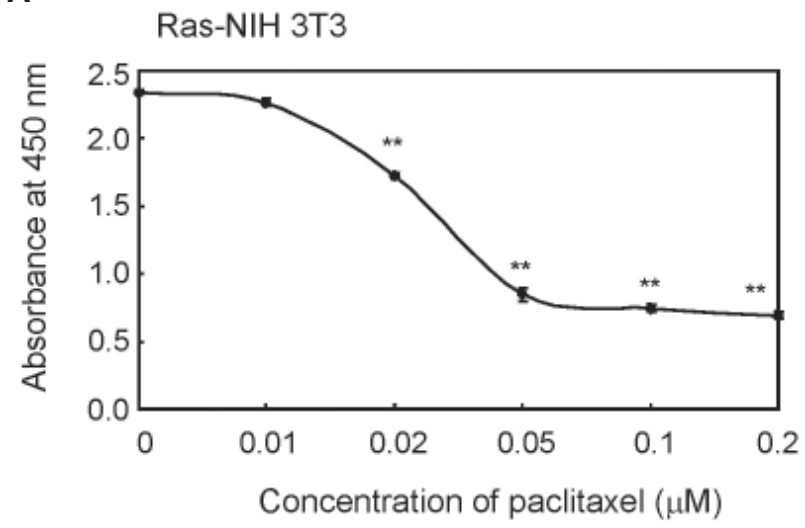

B

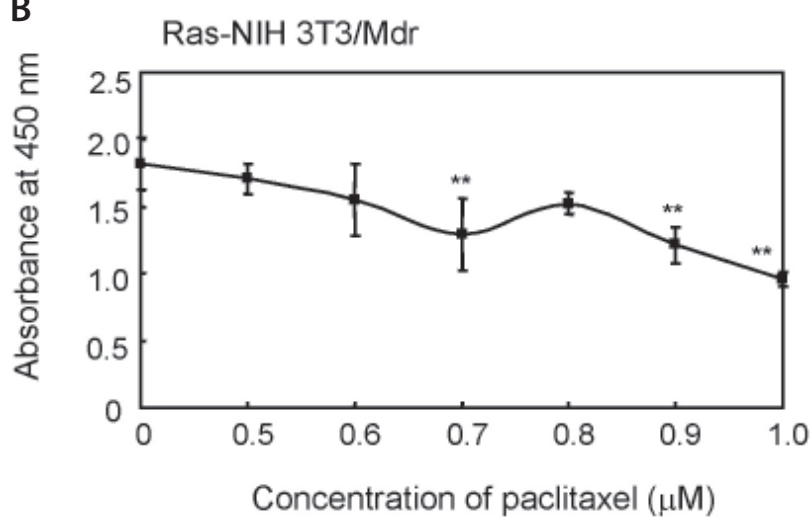

Fig. 1. The differential inhibitory effect of paclitaxel on Ras-NIH 3T3 (A) and Ras-NIH 3T3/Mdr cells (B). Each cell line was treated with increasing concentrations of paclitaxel and then incubated in 96 -well plates for the indicated days. The absorbance at $450 \mathrm{~nm}$ is expressed as the mean \pm SD of quadruplicate determinants from one of three representative experiments. ${ }^{* *} p<0.01$ and as determined by two-tailed test as compared to the mock control group.

for 2 hours at $4^{\circ} \mathrm{C}$, immunoprecipitates were washed three times with lysis buffer, and once with kinase buffer $(20 \mathrm{mM}$ Tris, $\mathrm{pH}$ 7.4, $20 \mathrm{mM} \mathrm{NaCl}, 1 \mathrm{mM}$ dithiothreitol, $10 \mathrm{mM} \mathrm{MgCl}$ ). Raf-1 kinase activity was measured by phosphorylation of recombinant MEK (Santa Cruz Biotechnology) as previously described (Lee, 2006). The washed immunoprecipitates were incubated in $40 \mu$ of kinase buffer containing $100 \mu \mathrm{M}$ ATP, and $1 \mu \mathrm{g}$ of the recombinant MEK at $30^{\circ} \mathrm{C}$ for $30 \mathrm{~min}$. The samples were resolved by SDS-PAGE, transferred to nitrocellulose membrane and probed with a polyclonal anti-phospho-MEK antibody. Detection was accomplished using the ECL-Plus chemiluminescent system and visualized using KODAK Image Station 4000 .

\section{Immunofluorescence staining}

For immunofluorescence experiments, cells were grown on chamber slides (Nunc), and fixed in 10\% formalin solution for $10 \mathrm{~min}$ as described (Ahn et al., 2010). Samples were blocked with blocking solution (1\% BSA, $0.6 \%$ Triton X-100 in PBS) for $1 \mathrm{~h}$ and incubated with primary antibodies diluted in blocking
A

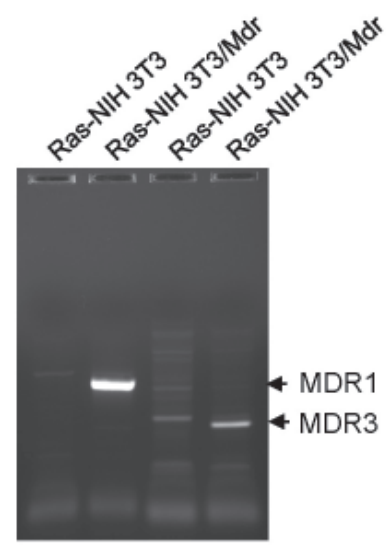

B

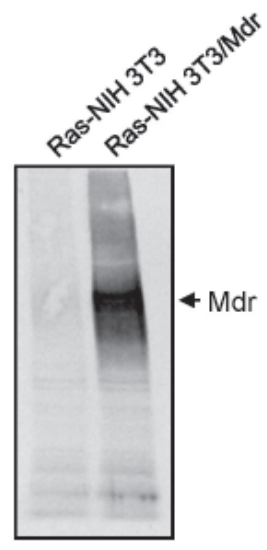

C

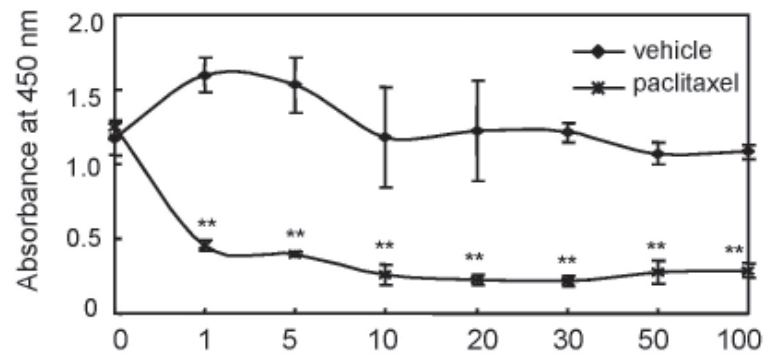

Fig. 2. Relative expression of MDR1 and MDR3 in Ras-NIH 3T3 and Ras-NIH 3T3/Mdr cells. (A) RT-PCR. The cDNA derived from total RNA starting material underwent 30 cycles of PCR. (B) Immunoblotting analysis. The expression of MDR was assessed by immunoblotting with anti-MDR antibody that detects both Mdr-1 and Mdr-3. The results presented are representative of at least three independent experiments. (C) To determine the effect of verapamil on paclitaxel cytotoxicity, increasing concentrations of verapamil with or without paclitaxel were added to Ras-NIH 3T3/Mdr cells. Cell growth inhibition was measured by the WST assay. The absorbance at $450 \mathrm{~nm}$ is expressed as the mean \pm SD of quadruplicate determinants from one of three representative experiments. ${ }^{* *} p<$ 0.01 as determined by Dunnett multiple comparison test as compared to the vehicle control group.

solution for $16 \mathrm{~h}$ at $4^{\circ} \mathrm{C}$. FITC-coupled anti-mouse IgG was used to detect Raf-1 proteins while Texas Red-coupled antirabbit IgG was used to visualize Spry2. Photographs were taken at 1000X magnification through a Zeiss Axio Scope.A1 epifluorescence microscope (Carl Zeiss Microlmaging, Inc., Thornwood, NY).

\section{Co-immunoprecipitation and immunoblot analysis}

The cells were transiently transfected with vector expressing FLAG-tagged wt-Raf-1 in combination with that expressing the full-length Spry2 using Lipofectamine 2000 (Invitrogen) according to the manufacturer's protocol. To isolate Spry2-Raf-1 complex, immunoprecipitation was performed on the whole cell lysates using monoclonal anti-FLAG M2, and protein A-agarose beads. For detection of Spry2 proteins coimmunoprecipitated with FLAG-Raf-1, immunoprecipitates were denatured in Laemmli sample buffer, and resolved by $10 \%$ SDS-polyacrylamide gel. The proteins were transferred to nitrocellulose, and immunoblot analysis was performed us- 
ing a polyclonal anti-Spry2. Immune complexes on nitrocellulose were detected by enzyme-linked chemiluminescence (Amersham Pharmacia Biotech, Piscataway, NJ). Fluorescent images were captured using KODAK Image Station 4000R.

\section{RESULTS}

\section{Establishment of paclitaxel-resistant cell lines, Ras-NIH 3T3/Mdr}

After establishment of paclitaxel-resistant Ras-NIH 3T3/ Mdr cell line by stepwise exposure to increasing concentrations of paclitaxel for 18 weeks, we determined paclitaxel sensitivity in Ras-NIH 3T3/Mdr and compared it with that of its parental Ras-NIH 3T3 cells. The IC ${ }_{50}$ for paclitaxel of Ras-NIH 3 T3 cells was less than $25 \mathrm{nM}$ (Fig. 1A). The WST-1 assay demonstrated that Ras-NIH 3T3/Mdr cells become 40 -fold $\left(\mathrm{IC}_{50}\right.$ : more than $\left.1 \mu \mathrm{M}\right)$ more paclitaxel resistant than Ras-NIH 3T3 cells (Fig. 1B). Additionally, they became resistant to other drugs too therefore a typical MDR phenotype was induced (data not shown).

\section{Drug resistance observed in Ras-NIH 3T3/Mdr is mediated through overexpression of the MDR gene}

The loss of the efficacy of anticancer therapy is correlated predominantly with the overexpression of P-glycoproteins that actively efflux chemotherapeutic drugs (Gottesman, 2002). MDR1 and MDR3 that belong to the ATP-binding cassettes $(A B C)$ family are well-known typical transporters. We evaluated the expression of MDR1 and MDR3 of Ras-NIH 3T3 and Ras-NIH 3T3/Mdr cells by RT-PCR analysis. MDR3 as well as MDR-1 mRNA was overexpressed in Ras-NIH 3T3/Mdr cells but not in Ras-NIH 3T3 cells (Fig. 2A). We also confirmed the increased expression of Mdr proteins in Ras-NIH 3T3/Mdr by immunoblotting analysis (Fig. 2B). Additionally, we examined whether verapamil affects cell proliferation in Ras-NIH 3T3/ Mdr cells. Verapamil, calcium channel blocker, has been re- ported to inhibit the transport function of P-glycoprotein (Nobili et al., 2006). Paclitaxel resistance of Ras-NIH 3T3/Mdr cells was completely blocked with the addition of non-cytotoxic concentrations of verapamil (Fig. 2C), suggesting that the overexpression of MDR mRNA in Ras-NIH 3T3/Mdr cells results in the production of a functional P-glycoprotein.

\section{The role of Raf-1 kinase in acquired multidrug resistance of Ras-NIH 3T3/Mdr cells}

One of the reported mechanisms by which of MDR is induced in paclitaxel-resistant cells is the induction by Raf-1 (Davis et al., 2003). As shown in Fig. 3A, Raf-1 kinase analysis revealed that basal Raf-1 kinase activity was higher in Ras$\mathrm{NIH}$ 3T3/Mdr cells than in Ras-NIH 3 T3 cells, suggesting that Raf-1 kinase activity in drug-resistant cells is closely correlated to the development of drug resistance. As shown in Fig. 3B, it was also observed significantly increased phosphorylation of downstream kinase of Raf-1, ERK, in Ras-NIH3T3/Mdr cells. Since the cell death by paclitaxel is associated with apoptosis, we also compared the expression of major apoptosis-related genes, Bcl-2, and cleaved PARP in these cells. However, expression level of all of these genes was not changed between parent and resistant cells (Fig. 3C).

\section{The change in the interaction between Raf-1 and Spry2 in Ras-NIH 3T3/Mdr cells}

Our recent data indicate that Spry2 may regulate Raf-1 kinase activity by acting as a scaffolding protein that brings Raf-1 kinase into close proximity with its direct regulators (Ahn et al., 2010). Here, we revealed that PDGF-induced Raf-1 activation was inversely correlated with the strength of interaction with Spry2 as monitored by co-immunoprecipitation experiment (Fig. 4A). Next, the endogenous expression levels of Spry2 were evaluated in both cell lines. The expression level of Spry2 in Ras-NIH 3T3/Mdr cells was much higher than in its parental cells (Fig. 4B). The Raf-1 association with Spry2 in intact cells was further investigated by immunofluores-

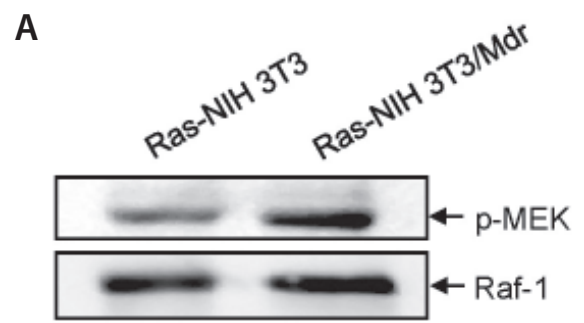

B

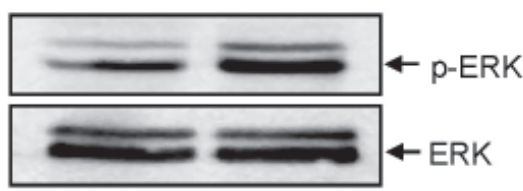

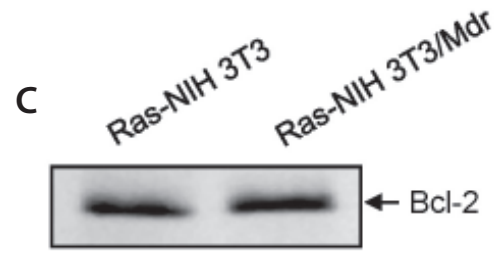

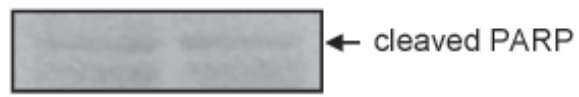

Fig. 3. The differential activation of Raf-1 kinase in Ras-NIH 3 T3 and Ras-NIH 3T3/Mdr cells. (A) In vitro Raf-1 kinase assays were performed using recombinant MEK-1 as substrate on the Raf-1 proteins immunoprecipitated from the whole cell lysates of both cell line. The same blot was stripped and then probed for Raf-1 to show similar expression level of each protein in all lanes. (B) The phosphorylated form of ERK was detected with immunoblotting using anti-phospho-ERK antibody. The same blot was stripped and then probed for ERK to show similar expression level of each protein in all lanes. (C) The expressions of two apoptosis-related genes, Bcl-2 and Poly (ADP-ribose) polymerase were assessed by immunoblotting with anti-Bcl-2 and anti-cleaved poly (ADP-ribose) polymerase antibody that detect cleaved products $(85 \mathrm{kDa})$. The results presented are representative of at least three independent experiments carried out under the conditions described. 
A

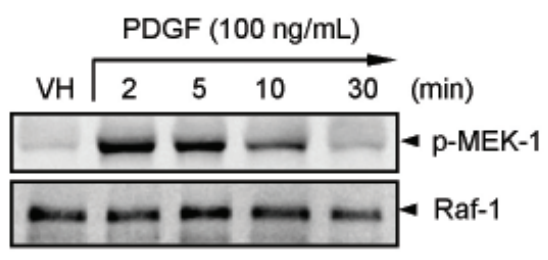

B

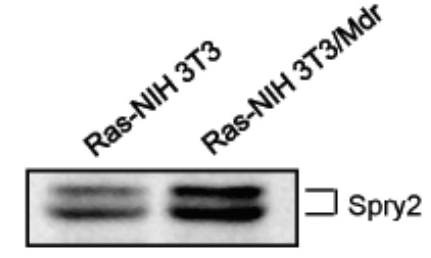

C

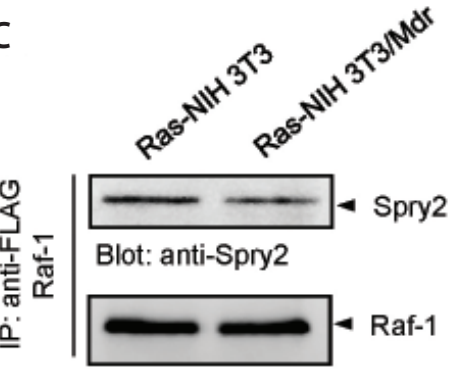

Blot: anti-Raf-1
PDGF $(100 \mathrm{ng} / \mathrm{mL})$

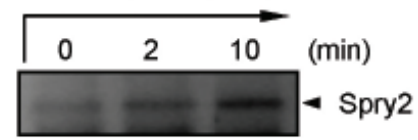

(IP, anit-Raf; Blot, anti-Spry2)

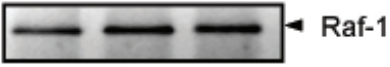

(IP, anit-Raf; Blot, anti-Raf)

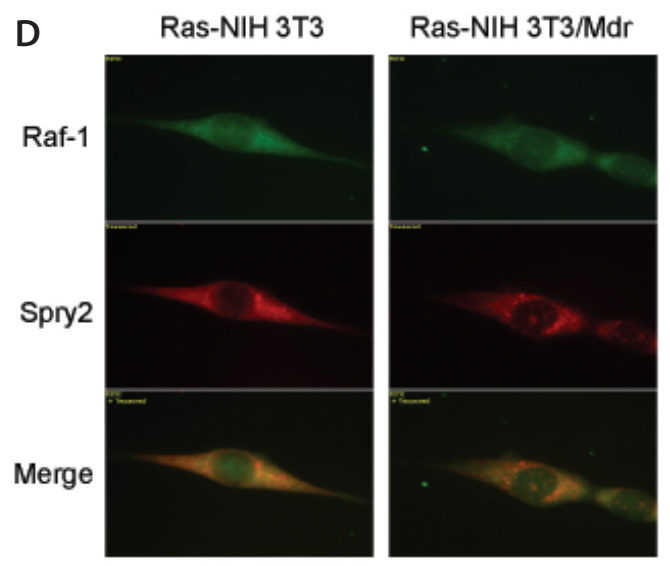

Fig. 4. The change in the interaction between Raf-1 and Spry2 in Ras-NIH 3T3/Mdr cells. (A) The role of Spry2 in PDGF-induced Raf-1 kinase activation. Subconfluent NIH 3T3 cells were serum-deprived for $24 \mathrm{~h}$ and then exposed to the $100 \mathrm{ng} / \mathrm{ml}$ of PDGF for the indicated time. The left panel, in vitro Raf-1 kinase assays were performed on the immunoprecipitated Raf-1 proteins using recombinant MEK-1 as a substrate. The right panel, Raf-1 proteins were immunoprecipitated from the whole cell lysates with anti-Raf-1 antibody. Aliquots of immunoprecipitates were analyzed by Western immunoblotting with anti-Spry2 antibody. The same blot was stripped and then probed for Raf-1 to show the expression level of Raf-1 in all lanes. (B) Cell extracts were prepared from Ras-NIH 3T3 and Ras-NIH 3T3/Mdr cells. The expression of Spry2 was examined by immunoblot analysis using a 1:1000 dilution of anti-Spry2 antibody. (C) Colocalization of overexpressed Spry2 with Raf-1 proteins. Both of Ras-NIH 3 T3 and Ras-NIH 3T3/Mdr cells were transiently transfected with vector expressing FLAGtagged Raf-1 in combination with vector expressing full-length Spry2. Twenty-four hours post-transfection the overexpressed FLAG-tagged Raf-1 mutants and Spry2 proteins were double immunostained with mouse monoclonal anti-FLAG antibody and rabbit polyclonal anti-Spry2 antibody, and subsequently reacted with goat anti-mouse IgG-FITC and bovine anti-rabbit IgG-Texas Red, respectively. Fluoresecence was captured by fluorescence microscopy. Green and red colors represent FLAG-Raf-1 and Spry2, respectively; yellow indicates colocalization of both proteins. The results presented are representative of at least three independent experiments performed under the conditions described. (D) FLAG-tagged Raf-1 mutants were immunoprecipitated with the anti-FLAG M2 antibody from the lysate of cells coexpressing Spry2 and Raf-1. Aliquots of the immunoprecipitates were analyzed by Western immunoblotting with the anti-Spry2 antibody. The same blot was stripped and then probed for Raf-1 to show expression level of Raf-1 in all lanes.

cence colocalization in Ras-NIH 3T3 and Ras-NIH 3T3/Mdr cells (Fig. 4C). Immunofluorescence studies revealed Raf-1 proteins was localized to the cytoplasm and nucleus in both cell lines. Spry2 proteins were cytoplasmic with concentrated localization around the nucleus in the perinuclear zone in Ras-NIH 3T3/Mdr cells whereas showing more diffused in the cytoplasm in Ras-NIH 3T3 cells. Merged images of Raf-1 and Spry2 showed colocalization in the cytoplasm of Ras-NIH 3T3 cells. However, the colocalization of Raf-1 and Spry2 was less observed in cytoplasm of Ras-NIH 3T3/Mdr cells due to concentrated localization of Spry2 around the nucleus in the perinuclear zone. In order to further confirm the change in the interaction of Raf-1 and Spry2, we tested the ability of Raf1 to interact with Spry2 in Ras-NIH 3T3/Mdr cells and their parental cells expressing FLAG-tagged Raf-1 (Fig. 4D). As expected, we observed that the interaction level of Raf- 1 and
Spry2 in Ras-NIH 3T3/Mdr cells were less than that in Ras$\mathrm{NIH}$ 3T3 cells. These observations led to the hypothesis that Raf-1 may be released from negative feedback inhibition by interacting with Spry2.

\section{Effect of RNAi targeting Raf-1 on chemosensitivity to paclitaxel in Ras-NIH 3T3/Mdr cells}

In order to investigate the hypothesis that Raf-1 kinase plays a positive role in drug resistance, we knocked down the Raf-1 mRNA by shRNA. We first tested the silencing efficiency of the shRNAs against endogenous Raf-1. Of those tested, shRNA\#1 was the most effective in decreasing the expression of Raf-1 (data not shown). Raf-1 shRNA transfection significantly decreased P-glycoprotein expression compared with controls as determined by western blot assay (Fig. 5A). 
A

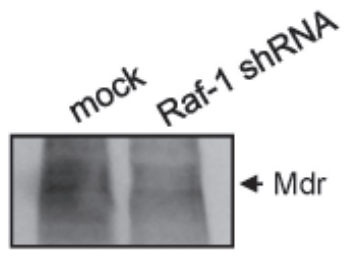

B

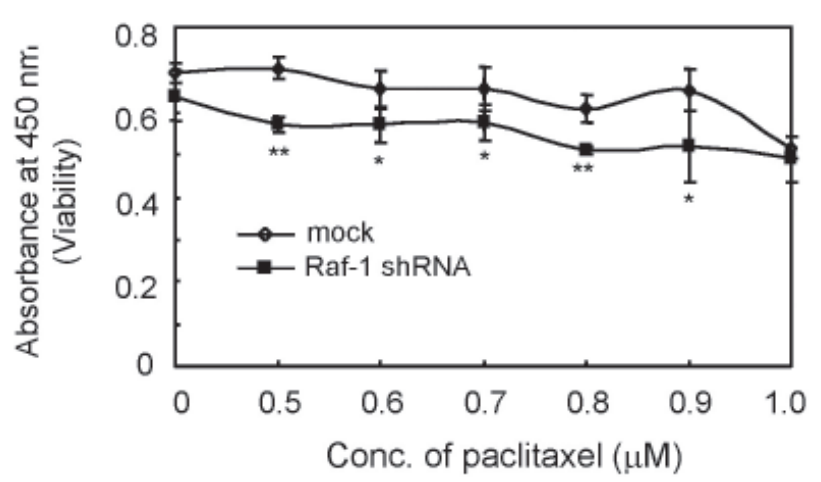

Fig. 5. Recovery of paclitaxel sensitivity by Raf-1 knockdown. In (A) and (B), the Ras-NIH 3T3/Mdr cells were transfected with Raf1 shRNA or a non-targeting control shRNA. (A) The expression of MDR was assessed by immunoblotting with anti-MDR antibody. The results presented are representative of at least three independent experiments. (B) The Raf-1 knockdown cells were treated with increasing concentrations of paclitaxel ranged from $0.5 \mu \mathrm{M}$ to 1.0 $\mu \mathrm{M}$, and then incubated in 96-well plates for 3 days. Cell growth inhibition was measured by the WST assay. Values represent the mean \pm SD of quadruplicate determinants from one of three representative experiments. ${ }^{* *} p<0.01$ and ${ }^{*} p<0.05$ as determined by two-tailed test as compared to the mock control group.

Next, WST-1 assay was employed to investigate the reversal effect of Raf-1 shRNA transfection on the multidrug resistance of Ras-NIH 3T3/Mdr cells. Fig. 5B shows that Raf-1 knockdown cells were more susceptible to paclitaxel treatment than mock cells. The partial rescue after Raf- 1 siRNA transfection could be explained by incomplete knockdown of Raf- 1 in the experiment. Actually, our preliminary data showed that $30 \%$ of Raf-1 still remained after Raf-1 siRNA transfection due to the incomplete knockdown of siRNA (data not shown). These results support a role for Raf-1 in mediating the drug resistance of Ras-NIH 3T3/Mdr cells.

\section{DISCUSSION}

The acquisition of multidrug resistance by cancer cells is correlated predominantly with the overexpression of P-glycoproteins that actively efflux chemotherapeutic drugs (Gottesman, 2002). Here, we also identified that verapamil, a specific P-glycoprotein efflux pump inhibitor (Nobili et al., 2006), completely restored paclitaxel sensitivity in Ras-NIH 3T3/Mdr cells, implying that $\mathrm{P}$-glycoprotein overexpression is the main reason of paclitaxel resistance in Ras-NIH 3T3/Mdr cell line. In addition, our results showed that one of mechanisms by which P-glycoprotein overexpression was induced in Ras- $\mathrm{NIH}$ 3T 3 cells was Raf- 1 kinase activation, indicating that the distal events triggered by Raf- 1 activation are the ultimate effectors of the drug resistance phenotype. Conversely, we could not detect differences of expression in apoptosis-related genes, such as bcl-2 and PARP between parental and paclitaxelresistant cell lines Our findings for the role of Raf- 1 kinase in drug-resistance are very consistent with other reports, suggesting that Raf-1 signal transduction pathways may be involved in the regulation of several aspects of drug resistance (Weinstein-Oppenheimer et al., 2001; Abrams et al., 2010). Several studies reported that increased activation of Raf-1 may up-regulate transcription of P-glycoprotein (Cornwell and Smith, 1993; Kim et al., 1996). In addition, there are several reports showing that modulation of Raf-1 kinase altered susceptibility of breast cancer cells to the chemotherapeutic drugs (Weinstein-Oppenheimer et al., 2001; Davis et al., 2003; Lee et al., 2003).

Although a correlation between high Raf-1 activity and drug resistance has been suggested in several reports (WeinsteinOppenheimer et al., 2001; Zhong et al., 2001), the induction mechanism for constitutively active Raf-1 kinase responsible for P-glycoprotein overexpression remains unclear. In contrast to the detailed data collected on the Raf-1 activation process, the mechanism responsible for Raf-1 inactivation after signaling events is much less understood. Possible candidate for the feedback negative regulation of the Raf-1/MAP kinase pathway is the Sprouty (Spry) protein (Yusoff et al., 2002; Sasaki et al., 2003; Ahn et al., 2010), which was found to be a general inhibitor of receptor tyrosine kinases (Hacohen et al., 1998). Our study revealed that the expression level of Spry2 in Ras-NIH 3T3/Mdr cells was much higher than in their parental cells. Unexpectedly, however, immunofluorescence assay showed that colocalization of Raf-1 and Spry2 was less observed in cytoplasm of Ras-NIH 3T3/Mdr cells compared to Ras-NIH 3T3 cells due to concentrated localization of Spry2 around the nucleus in the perinuclear zone. These observations led to the hypothesis that Raf- 1 might be released from negative feedback inhibition by interacting with Spry2 in RasNIH 3T3/Mdr cells.

Although Raf-1 overexpression correlated with the development of multidrug resistance in Ras-NIH 3T3/Mdr cells, RNAi targeting Raf-1 partially restored chemosensitivity to paclitaxel in Ras-NIH 3T3/Mdr cells. Hyperactivation of Raf-1 kinase may not be sufficient as an explanation of the mechanism that Ras-NIH 3T3/Mdr cells acquire the multidrug-resistance phenotype after exposure to paclitaxel. Mercer and Pritchard (2003) identified B-Raf rather than Raf-1 as the primary Raf isoform for for activating ERK pathway. Thus, future studies are aimed at elucidating the molecular mechanisms underlying the biological effects of B-Raf on acquired multidrug resistance. This will, in turn, help us understand B-Raf modulation through negative feedback regulation of Sprouty by paclitaxel.

\section{ACKNOWLEDGMENTS}

This work was supported by the University of Incheon Research Grant in 2010.

\section{REFERENCES}

Abrams, S. L., Steelman, L. S., Shelton, J. G., Wong, E. W., Chap- 
pell, W. H., Basecke, J., Stivala, F., Donia, M., Nicoletti, F., Libra, M., Martelli, A. M. and McCubrey, J. A. (2010) The Raf/MEK/ERK pathway can govern drug resistance, apoptosis and sensitivity to targeted therapy. Cell Cycle 9, 1781-1791.

Ahn, J. H., Eum, K. H. and Lee, M. (2009) The enhancement of Raf-1 kinase activity by knockdown of Spry2 is associated with high sensitivity to paclitaxel in v-Ha-ras-transformed NIH 3T3 fibroblasts. Mol. Cell. Biochem. 332, 189-197.

Ahn, J. H., Eum, K. H. and Lee, M. (2010) Spry2 does not directly modulate Raf-1 kinase activity in v-Ha-ras-transformed NIH 3T3 fibroblasts. BMB Rep. 43, 205-211.

Blobe, G. C., Sachs, C. W., Khan, W. A., Fabbro, D., Stabel, S., Wetsel, W. C., Obeid, L. M., Fine, R. L. and Hannun, Y. A. (1993) Selective regulation of expression of protein kinase $C(P K C)$ isoenzymes in multidrug-resistant MCF-7 cells. Functional significance of enhanced expression of PKC alpha. J. Biol. Chem. 268, 658-664.

Callaghan, R. and Higgins, C. F. (1995) Interaction of tamoxifen with the multidrug resistance P-glycoprotein. Br. J. Cancer 71, 294-299.

Chatterjee, D., Bai, Y., Wang, Z., Beach, S., Mott, S., Roy, R., Braastad, C., Sun, Y., Mukhopadhyay, A., Aggarwal, B. B., Darnowski, J., Pantazis, P., Wyche, J., Fu, Z., Kitagwa, Y., Keller, E. T., Sedivy, J. M. and Yeung, K. C. (2004) RKIP sensitizes prostate and breast cancer cells to drug-induced apoptosis. J. Biol. Chem. 279, 1751517523.

Cornwell, M. M. and Smith, D. E. (1993) A signal transduction pathway for activation of the mdr1 promoter involves the proto-oncogene c-raf kinase. J. Biol. Chem. 268, 15347-15350.

Davis, J. M., Navolanic, P. M., Weinstein-Oppenheimer, C. R., Steelman, L. S., Hu, W., Konopleva, M., Blagosklonny, M. V., McCubrey, J. A. (2003) Raf-1 and Bcl-2 induce distinct and common pathways that contribute to breast cancer drug resistance. Clin. Cancer Res. 9, 1161-1170.

Dong, Y., Shao, S., Hu, J. and Yang, P. (2009) Reversal effect of Raf-1/ Mdr-1 siRNAs co-transfection on multidrug resistance in KBv200 cell line. Oral Oncol. 45, 991-997.

Gottesman, M. M. (2002) Mechanisms of cancer drug resistanace. Annu. Rev. Med. 53, 615-627

Gupta, K. P., Ward, N. E., Gravitt, K. R., Bergman, P. J. and O'Brian, C. A. (1996) Partial reversal of multidrug resistance in human breast cancer cells by an $\mathrm{N}$-myristoylated protein kinase $\mathrm{C}$-alpha pseudosubstrate peptide. J. Biol. Chem. 271, 2102-2111.

Hacohen, N., Kramer, S., Sutherland, D., Hiromi, Y. and Krasnow, M. A. (1998) Sprouty encodes a novel antagonist of FGF signaling that patterns apical branching of the Drosophila airways. Cell 92 253-263.

Haldar, S., Chintapalli, J. and Croce, C. M. (1996) Taxol induces bcl-2 phosphorylation and death of prostate cancer cells. Cancer Res. 56, 1253-1255.

Jaffrezou, J. P., Herbert, J. M., Levade, T., Gau, M. N., Chatelain, P. and Laurent, G. (1991) Reversal of multidrug resistance by calcium channel blocker SR33557 without photoaffinity labeling of P-glycoprotein. J. Biol. Chem. 266, 19858-19864.

Jazirehi, A. R., Vega, M. I., Chatterjee, D., Goodglick, L. and Bonavida, B. (2004) Inhibition of the Raf-MEK1/2-ERK1/2 signaling pathway, $\mathrm{Bcl}-\mathrm{xL}$ down-regulation, and chemosensitization of non-Hodgkin's lymphoma B cells by Rituximab. Cancer Res. 64, 7117-7126.
Kim, S. H., SH, L., NH, K., CD, K. and BS, C. (1996) Effect of the activated Raf protein kinase on the human multidrug resistance 1 (MDR1) gene promoter. Cancer Lett. 98, 199-205.

Lee, L. F., Haskill, J. S., Mukaida, N., Matsushima, K. and Ting, J. P. (1997) Identification of tumor-specific paclitaxel (Taxol)-responsive regulatory elements in the interleukin-8 promoter. Mol. Cell. Biol. 17, 5097-5105.

Lee, M. (2006) Raf-1 kinase activation is uncoupled from downstream MEK/ERK pathway in cells treated with Src tyrosine kinase inhibitor PP2. Biochem. Biophys. Res. Commun. 350, 450-456.

Lee, M., Ahn, J. H. and Eum, K. H. (2009) The differences in biological properties between parental and v-Ha-ras transformed NIH3T3 cells. Cancer Res. Treat. 41, 93-99.

Lee, M., Koh, W. S. and Han, S. S. (2003) Down-regulation of Raf1 kinase is associated with paclitaxel resistance in human breast cancer MCF-7/Adr cells. Cancer Lett. 193, 57-64.

Marshall, M. S. (1995) Ras target proteins in eukaryotic cells. FASEB J. 9, 1311-1318.

McGrogan, B. T., Gilmartin, B., Carney, D. N. and McCann, A. (2008) Taxanes, microtubules and chemoresistant breast cancer. Biochim. Biophys. Acta 1785, 96-132.

Mercer, K. E. and Pritchard, C. A. (2003) Raf proteins and cancer: B-Raf is identified as a mutational target. Biochim. Biophys. Acta 1653, 25-40.

Nobili, S., Landini, I., Giglioni, B. and Mini, E. (2006) Pharmacological strategies for overcoming multidrug resistance. Curr. Drug Targets 7, 861-879.

Sasaki, A., Taketomi, T., Kato, R., Saeki, K., Nonami, A., Sasaki, M., Kuriyama, M., Saito, N., Shibuya, M. and Yoshimura, A. (2003) Mammalian Sprouty4 suppresses Ras-independent ERK activation by binding to Raf1. Nat. Cell Biol. 5, 427-432.

Weinstein-Oppenheimer, C. R., Henríquez-Roldán, C. F., Davis, J. M., Navolanic, P. M., Saleh, O. A., Steelman, L. S., Franklin, R. A., Robinson, P. J., McMahon, M. and McCubrey, J. A. (2001) Role of the Raf signal transduction cascade in the in vitro resistance to the anticancer drug doxorubicin. Clin. Cancer Res. 7, 2898-2907.

Wu, C. P., Calcagno, A. M. and Ambudkar, S. V. (2008) Reversal of $A B C$ drug transporter-mediated multidrug resistance in cancer cells: evaluation of current strategies. Curr. Mol. Pharmacol. 1, 93105.

Yeung, K., Seitz, T., Li, S., Janosch, P., McFerran, B., Kaiser, C., Fee, F., Katsanakis, K. D., Rose, D. W., Mischak, H., Sedivy, J. M. and Kolch, W. (1999) Suppression of Raf-1 kinase activity and MAP kinase signalling by RKIP. Nature 401, 173-177.

Yusa, K. and Tsuruo, T. (1989) Reversal mechanism of multidrug resistance by verapamil: direct binding of verapamil to P-glycoprotein on specific sites and transport of verapamil outward across the plasma membrane of K562/ADM cells. Cancer Res. 49, 5002-5006.

Yusoff, P., Lao, D. H., Ong, S. H., Wong, E. S., Lim, J., Lo, T. L., Leong, H. F., Fong, C. W. and Guy, G. R. (2002) Sprouty2 inhibits the Ras/MAP kinase pathway by inhibiting the activation of Raf. J. Biol. Chem. 277, 3195-3201.

Zhong, J., Troppmair, J. and Rapp, U. R. (2001) Independent control of cell survival by Raf-1 and Bcl-2 at the mitochondria. Oncogene 20, 4807-4816. 